\title{
The Effects of Anti-Fibrosis Drugs on the Self- Regulation of Alveolar Macrophages and Interstitial Macrophages in the Pathological Process of Pulmonary Fibrosis
}

\author{
Huaqiang Zhai ${ }^{1 *}$, Guoxiu Liu ${ }^{1}$, Siyu Li ${ }^{1}$, Ningning Li ${ }^{1}$, Ying Dai ${ }^{1}$, Ningning Wang ${ }^{1}$, Yiping Yuan ${ }^{1}$, Zhaojuan Guo ${ }^{1}$, \\ Tian Zhang ${ }^{2}$ and Hongyan $\mathrm{Li}^{3}$
}

${ }^{1}$ School of Chinese Pharmacy, Beijing University of Chinese Medicine, China

${ }^{2}$ Department of pharmacy, Beijing Hospital, China

${ }^{3}$ Department of pharmacy, China

Submission: February 13, 2018; Published: September 10, 2018

"Corresponding author: Huaqiang Zhai, School of Chinese Pharmacy, Beijing University of Chinese Medicine, No.11, North third ring road, Chaoyang District, Beijing, 100029, China, Tel: +86 010 84738630, Fax: +86 010 84738611; Email: jz711@qq.com

\section{Abstract}

Pulmonary fibrosis is a devastating condition with many kinds of inducements. Alveolar macrophages (AMs) and interstitial macrophages (IMs) are two subsets of macrophages which involve in the inflammatory reaction of pulmonary fibrosis. Traditional Chinese medicine has good effects on the treatment of pulmonary fibrosis. This review is aimed at summarizing the different roles of alveolar macrophages and interstitial macrophages in the pathological process of pulmonary fibrosis progression and anti-fibrosis drugs related to the self-regulation mechanism of these two kinds of cells, furthermore to explain the multiple mechanisms of traditional Chinese medicine for treating pulmonary fibrosis from the self-regulation of macrophages. Searches for keywords "alveolar macrophages", "interstitial macrophages", "pulmonary fibrosis" and "IPF" were performed in biomedical databases. The reported incidences of AMs and IMs in the pathological process of pulmonary fibrosis are involved in this article, and the influences of traditional Chinese medicine on the cells are summarized.

A comprehensive analysis of the literature confirms that alveolar macrophages appear to be better equipped for their antimicrobial task, whereas interstitial macrophages have more capacity for immune regulatory functions. Disease-modifying therapy for pulmonary fibrosis based on intervening the macrophages self-regulation relates to chemokines and cytokines. Traditional Chinese herbs for pulmonary fibrosis have different targets on the two kinds of cells. Alveolar macrophages and interstitial macrophages could be used to explore the pathological process and clinical medicine of pulmonary fibrosis, and they are conducive to interpret the multiple mechanisms of TCM for pulmonary fibrosis.

Keywords: Alveolar macrophages; Interstitial macrophages; Pulmonary fibrosis; Chemokines; Cytokines; Traditional Chinese medicine

Abbreviations: AMs: Alveolar Macrophages; IMs: Interstitial Macrophages; IPF: Idiopathic Pulmonary Fibrosis; TCM: Traditional Chinese Medicine; BALF: Bronchoalveolar Lavage Fluid; PDGF: Platelet-Derived Growth Factor; TNF- $\alpha$ : Tumor Necrosis Factor-A; IFN: Interferon; TGF- $\beta 1$ : Transforming Growth Factor-B1; CTGF: Connective Tissue Growth Factor

\section{Introduction}

Pulmonary fibrosis is a kind of chronic progressive interstitial lung disease. Idiopathic pulmonary fibrosis (IPF) is a common and devastating pulmonary fibrosis with no powerful drugs. Data showed the incidence of IPF seems to be increasing rates of 3-9 cases per 100000 a year, although varies worldwide [1]. The median survival was 2.5 to 3.5 years after the diagnosis of IPF [2]. The major clinical features of pulmonary fibrosis are symptoms of dyspnea, wheezing, dry cough, etc. and eventually leading to respiratory failure and death [3]. Current researches about pulmonary fibrosis more focus on anti-inflammatory, immunity, cytokines, genetic factors, etc. [4]. A small number of experiments were conducted at the level of molecules and gene [5-7]. The etiology of pulmonary fibrosis is varied, with an array of triggers including allergens, chemicals, radiation and environmental particles [4]. Such triggers are believed to impair the tight regulation of inflammation and repair, leading to excess 
production of collagen by fibroblasts and the subsequent formation of scar tissue [8]. However, the cause of IPF remains unclear [9].

It is vital that tissue architecture is restored to regain normal function. The early lesions of pulmonary fibrosis mainly appear in the alveolar walls accompanied by a large number of proliferated lung fibroblasts, causing extracellular matrix deposits in alveolar and interstitial excessively [10]. Then the repaired fibrous tissues result in structural disorder and replace the normal tissues [11]. The ultimate outcome is irreversible pulmonary fibrosis $[12,13]$. An important factor of pulmonary fibrosis occurrence is the function and number of AMs and IMs [14]. Analysis of the different roles of AMs and IMs in the pathological process of pulmonary fibrosis in the field will provide an explanation of the multiple mechanisms for treating pulmonary fibrosis with TCM.

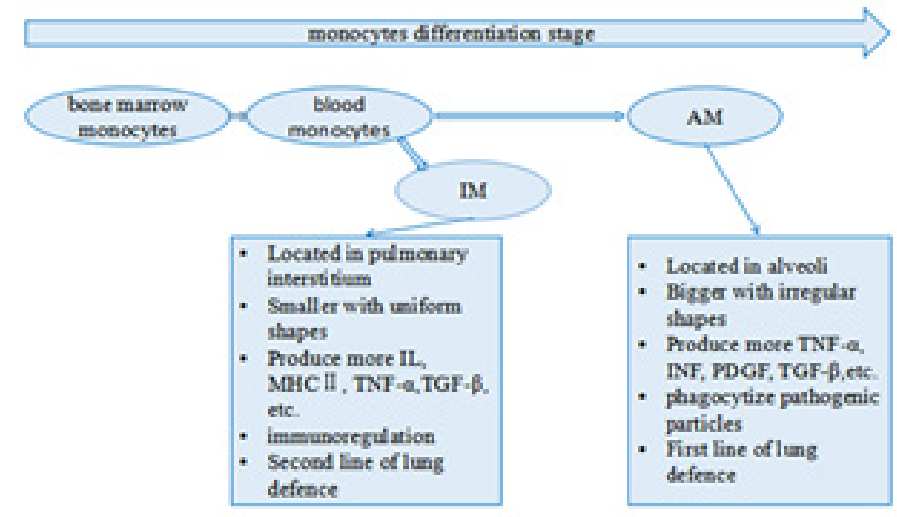

Figure 1: The monocytes differentiation stage and characters of AMs and IMs in pulmonary fibrosis.

Roles of AMs and IMs in the Pathological Process of Pulmonary Fibrosis

AMs and IMs are getting more and more attention in the treatment of pulmonary fibrosis. The basic functions of macrophages in the immune defence system are phagocytic and bactericidal activities, which is vital in resisting the invasion of pathogenic microorganisms. It is reported that AMs appeared to be better equipped for their antimicrobic task. Whereas IM, although also having antimicrobic potential, show a more pronounced capacity of immunoregulatory functions [15]. As the sole cell population exposed to air, AMs having phagocytic activity of clearing extraneous particles, which is the first barrier of the lung. AMs get damaged after phagocytizing the pathogenic particles, further cause lung damages. IMs in the lung tissue also play a very important role in inflammation and local immune defense reaction of the lung injury, which is the second barrier of the lung (Figure 1).

\section{The form and number of AMs and IMs in the pathologi- cal process of pulmonary fibrosis}

AMs and IMs are two critical subsets of macrophages. Pulmonary macrophages consist of AMs in the surface and internal of alveolar space, and IMs in pulmonary interstitial next to alveolar septa or next to bronchi and vessels. Normally, there are significant differences between AMs and IMs in morphology, structure, phenotype and function [16]. Under the light microscope, the diameter of AM is about $(12.1 \pm 1.16) \mu \mathrm{m}$ which is larger than that of IM with about $(9.3 \pm 1.1) \mu \mathrm{m}$. AM is round or irregular while IM is round and uniform relatively in shape and size. AM HE staining shows the cytoplasm is pink with loose nucleus deviate to the side of the cytoplasm in an oval shape and the nuclear-cytoplasmic ratio is less than $50 \%$. The surface of the AM is uneven with many protrusions and the cells contain more intracellular lysosomes. IM, HE is staining shows that the cytoplasm is dark red, with dense nucleus shape irregularly and the nuclear-cytoplasmic ratio is greater than $50 \%$ [17].

The surface of the IM is smooth with almost no protrusions and the cells contain fewer primary and secondary intracellular lysosomes. Differences between AMs and IMs in morphology and structure determine their differences in functions [18]. IMs only accounts for $3 \%-5 \%$ of the total number of various cells throughout the lung tissue, so the isolation of IM is totally a difficult task. Over $90 \%$ of the rat bronchoalveolar lavage fluid (BALF) samples are AMs, and a variety of cells including macrophages, neutrophils, endothelial cells and lymphocytes can be obtained by enzymatic dispersal of the lung tissue. Separated by the lymphocyte separation liquid and purified by adherent cell culture, $90 \%$ of the cells in culture liquid are IM cells. The number of AMs and IMs in lung tissues under normal physiological conditions is less than that of fibrosis pathological conditions. Studies have found that the number of AMs in BALF reached the highest level on the $7^{\text {th }}$ day after bleomycin injection, and the number and HE stain intensity of positive AMs have reached its peak as well [19]. And AMs increased significantly in the BALF on the $14^{\text {th }}$ and $30^{\text {th }}$ day after Pingyangmycin injection, which is consistent with the pro-inflammatory and 
pro-fibrotic effect of AMs. The number of AMs on the $14^{\text {th }}$ day of Pingyangmycin injection is more than that on the $30^{\text {th }}$ day, which may relate to the serious inflammatory injury during the first $14^{\text {th }}$ days. The number of AMs will continue to develop on the $30^{\text {th }}$ day with the sustained progress of pulmonary fibrosis [20].

\section{The cell function of AMs and IMs in the pathological} process of pulmonary fibrosis

AMs and IMs in the lung are distributed in two different anatomical sites, which result in their different functions $[21,22]$. Located in the airways and alveoli, AMs are the only cell population exposed to air, which contributes to the first barrier of the lung to clear inhaled microorganisms during breathing [23]. The morphology of the permanent AMs in alveoli is changeable, while the AMs in normal lung tissues are similar to the mature macrophages of other organizations. The microvilli of normal AMs distribute on the cell surface uniformly. The cytoplasmic volume of normal AMs is large with a large number of lysosomes, phagosomes and enzymes [24]. Besides, normal AMs produce cell recruitment factors, active nitrogen and other factors, and result in macrophages and other inflammatory cells gathering. During acute inflammatory periods, AMs become larger and containing quantities of peroxidase with a diameter of $12 \mu \mathrm{m}$, while they become mature and larger during chronic progress period with a diameter of $14-40 \mu \mathrm{m}$. The cytoplasmic membrane of AMs under the electron microscope is irregular.

The cytoplasm contains many mitochondria and lysosomes, and the nucleus is more leaflike. The expression and release of membrane receptors in AMs depend on different states. The main role of AMs is to kill pathogenic microorganisms, phagocytize water-soluble poorly organic particles, release inflammatory mediators, present antigens, and express different cytomembrane receptors at the same time. Many studies suggest that AMs are the key cells causing early biological effects on lung [25]. In the initial process of lung injury, cellular infiltration can also be found in AMs from the observation of lung tissue by biopsy. The degree of cellular infiltration is closely related to the degree of pulmonary fibrosis, which confirms that AMs have the function of promoting the inflammatory response. In the process of promoting inflammatory infiltration, it also increases lung damage and causes excessive repair of the tissues, then leads to fibrosis.

IMs also play a very important role in lung inflammation and local immune defence reaction. The IMs are a type of macrophages in the interstitial connective tissue forming the second line of the lung defence, which may have a greater immune function with its special location. But the location of IM can also make it difficult in extracting the cells. IMs in pulmonary interstitial contact with the extracellular matrix and other interstitial cells tightly thus affects pulmonary interstitial metabolism. IMs are often acquired from the pulping lung tissue digested by collagenase, but the separation methods reported in the literature are quite different. IMs have their own unique function and morphology compared with AMs. They have a smaller diameter of $6.6 \mu \mathrm{m}$, a more folded cell membrane, irregular nucleus and larger nucleoplasm ratio. And the non-specific lipid stain results of them can be positive, less positive or negative.

The filar pseudopod of IMs cell can be seen under the electron microscope. The cytoplasmic membrane of IMs is pleated, and still not extended to its characteristics after cultivating for $24 \mathrm{~h}$. Because of the difficulty in getting IMs, more papers focus on the study of AMs. However, recent studies have shown that IMs may have a more important role in lung injury. As the important cells, IM's ability to phagocytize particles, produce oxygen radicals and chemotactic complement is weaker than that of AM [15]. But IMs express more MHC II and they are more efficient in stimulating the proliferation of T cells [26].

The phagocytosis and secretory function of IMs are like that of AMs. Although the Fc receptor-dependent cellular phagocytosis capability of IMs is like that of AMs, the Fc receptor-independent cellular phagocytosis capability of IMs is lower than that of AMs significantly. So that IMs show more capable of respiratory immune responses. Existed in pulmonary interstitial, IMs can release inflammatory mediators and cytotoxic mediators, which may be a more direct cause of lung tissue damage [27]. There has also been reported that IMs make more sense in the progression of pulmonary fibrosis than AMs in biological behaviours and active effects [28]. More experiments should be carried out to confirm the function of IMs.

\section{The relationship between AMs and IMs in the patholo- gical process of pulmonary fibrosis}

AMs and IMs are two critical subsets of macrophages, which are critical in the pathological process of pulmonary fibrosis process. From the perspective of cells occurrence, AMs are the ultimate development status of macrophages which come from the source of bone marrow monocytes. Blood monocytes grew into AMs in the development of end-stage, while IMs may be the transitional stage of that process [18]. But there are great differences between AMs and IMs in antigen presenting, cytokines secreting and other immune functions. And the effects of endotoxin attacks on them are discrepant. All the differences suggest IMs are not simply the precursor of AMs.

Evidence also shows that platelet-derived growth factor (PDGF) secreted by AMs cannot enter the alveolar interstitial through the epithelia completely. Therefore, it may be concluded that AMs located in the alveolar may have a stronger function of phagocytosis, and IMs in the interstitial are primarily related to the immune regulation. In vitro studies, Adamson found that short fibers $(<1 \mu \mathrm{m})$ and long fibers $(>20 \mu \mathrm{m})$ asbestos could stimulate macrophages to produce the Fibroblast Growth cytokines. In vivo studies also found that AMs phagocytized almost all short fibers which did not enter the pulmonary interstitial without pulmonary fibrosis lesion when mice inhaled short asbestos fibers. While IMs were activated when the long fiber deposited 
on the bronchi furthered into the pulmonary interstitial and caused effectors accumulate in pulmonary interstitial. Then fibroblast multiplied quickly, eventually led to the formation of pulmonary fibrosis [29].

\section{The Self-Regulation Mechanism of AMs and IMs in the Pathological Process of Pulmonary Fibrosis}

Pulmonary fibrosis is characterized by the accumulation of fibroblasts. The deposition of inflammatory cells such as macrophages, lymphocytes, and granulocytes are also the hallmark of pulmonary fibrosis. The mechanisms responsible for the migration of fibroblasts and inflammatory cells to the lung in pulmonary fibrosis are not known, but cytokines and chemokines are essentially considered [30]. The self-regulation mechanism of AMs and IMs includes two parts: Firstly, the inflammatory factors stimulate macrophages and other immune cells to secrete chemokines [31], which allow AMs, IMs and other inflammatory cells to accumulate and release a large number of cytokines. Secondly, the release of cytokines stimulates myofibroblast, leading to the accumulation, proliferation and activation of fibroblasts, then pulmonary fibrosis occurs. By further activating signal transduction pathways at the same time, cytokines stimulate inflammatory cells to generate new cytokines and the process cycles [32] (Figure 2).

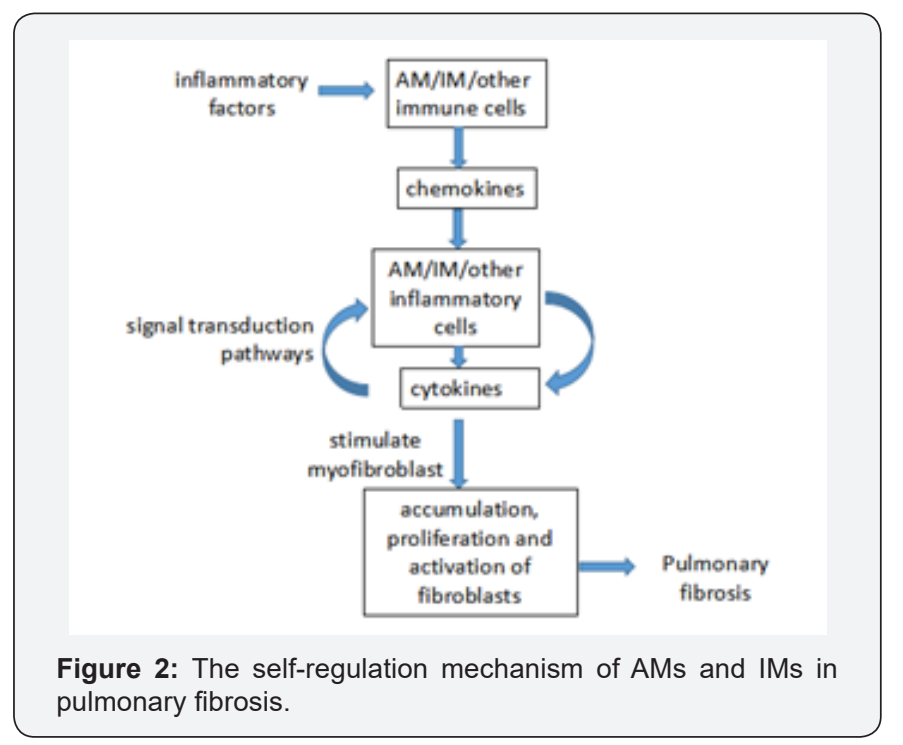

\section{Chemokines assist in the migration and invasion abili- ty of AMs and IMs}

The CC family and CXC family are two big families of chemokines involving the pulmonary fibrosis. The transfer of fibroblasts and inflammatory cells in the lung requires the participation of various chemokines/chemokine receptors such as CXCL12/CXCR4, CCL21/CCR7, CCL2/CCR2, CCL3/CCR5 [32]. Many biological functions of macrophages play their roles in their cytomembrane mediated action. The cytomembrane receptors of AMs and IMs correspond with their specific binding ligand, triggering the cell signalling pathways. The binding force of the receptor on the cell surface and the number of receptors on the surface of the cytomembrane can affect the signal transduction $[33,34]$. Phagocytosis is one of the important functions of macrophages, and the interaction between effector cells and the membrane of the target cells is a key factor in macrophages to kill tumor cells [35].

Cell migration is an important reason for adjusting the number of cells. Monocyte chemoattractant protein-1 (MCP1) and macrophage inflammatory protein-1 $\alpha$ (MIP-1 $\alpha$ ) (also known as CCL2 and CCL3) in-vivo are important chemokines participated in the chemotaxis of the cells, the main chemotactic cells and macrophages are concentrated in the inflammatory regions [36]. Studies have found that inhibiting the activity of MCP- 1 and MIP- $1 \alpha$ in the early pulmonary fibrosis can reduce the accumulation of alveolar macrophages [37]. MCP-1mRNA in the lung tissue of rats can be found mainly expressed in alveolar macrophages, airway epithelial cells in Bleomycin-induced pulmonary fibrosis rats [38]. Thus, MCP- 1 alters the population of alveolar macrophages through recruitment of blood monocytes into the luminal airspace. MIP- $1 \alpha$ can also mobilize the bone marrow monocytes into myeloid precursor cells and enhance the infiltration of macrophages in inflammation areas [39].

According to the general reasoning, the reason for the increase of AMs in the alveoli and IM in the pulmonary interstitial is likely to be under the chemotaxis of chemokines, blood monocytes cells infiltrate or migrate to the inflammatory sites and grow into the macrophages. The increased extent of AMs varies depending on the stage of the pulmonary fibrosis, while IMs increase more obviously in the early period. In vitro study of the macrophages migration and invasion chemotaxis of MCP-1 shows that MCP- 1 can assist in the migration and invasion ability of macrophages. And the chemotactic efficiency of macrophages related to the concentration of MCP-1 [40]. The growing number of macrophages with enhanced invasion capability can contribute to the pathological process of pulmonary fibrosis.

\section{AMs and IMs secrete cytokines to regulate fibroblasts and regulate cytokines secretion}

Macrophages are gathered and activated under the chemotaxis of chemokines. AMs secrete platelet-derived growth factor (PDGF) and transform growth factor beta (TGF- $\beta$ ), which can promote the accumulation, proliferation and activation of fibroblasts in lung injury area, causing the extracellular matrix accelerating the synthesis. It can also increase fibroblast by increasing the transfer and adhesion of osteopontin, resulting in pulmonary fibrosis [41]. AMs secrete tumor necrosis factor- $\alpha$ (TNF- $\alpha$ ), interferon (IFN), transforming growth factor- $\beta 1$ (TGF- $\beta 1$ ) and other active media that can anti-pathogenic microorganisms strongly, which can effectively kill the pathogens invaded the body. IMs have lower ability to produce these cytokines than AMs, while the ability to secrete cytotoxic medium and interleukin (IL) are high, which cause more direct damages to lung tissue and show higher MHCII class antigen expression activity and stronger supplementary ability. 
Cytokines regulate fibroblasts and macrophages involved in a variety of signal transduction pathways such as the MAPK pathway, JAK-STAT pathway, Smad pathway, PI3K-Akt pathway, NF- $\kappa B$ pathway, etc [42]. TGF- $\beta 1$, a powerful fibrosis cytokine, rely on Smads and MAPK signal transduction pathway to regulate lung fibroblasts to muscle fibroblasts, produce a large number of extracellular matrix proteins, and lead to pulmonary fibrosis [43]. IL, TGF- $\beta 1$, IFN involved in the JAK-STAT pathway to regulate the proliferation of fibroblasts by regulating the gene expression. At the same time, cytokines further activate the signal transduction pathways and stimulate AMs and IMs to produce new cytokines. TNF- $\alpha$ stimulates MAPK/ERK pathway, increases transcription of the TGF- $\beta 1$ Gene and stabilizes TGF- $\beta 1 \mathrm{mRNA}$ to induce the expression of TGF- $\beta 1[44,45]$. On the other hand, TNF- $\alpha$ activates NF- $\kappa$ B pathway to accelerate the new TNF- $\alpha$ secretion of macrophages [46]. The upgraded cytokines create new biological effects.

\section{Disease-Modifying Therapy for Pulmonary Fibrosis Based on Intervening the Self-Regulation of AMs and IMs}

Clinical medicinal therapies of pulmonary fibrosis including symptom-focused therapy and disease-modifying therapy. Randomised controlled trials show that various therapies (eg, prednisolone and azathioprine, acetylcysteine, and warfarin) were ineffective or harmful, but disease-modifying therapies with nintedanib and pirfenidone are effective [47]. Both drugs are currently approved worldwide. Thus, therapies focusing on modifying pulmonary fibrosis are promising.

AMs and IMs primarily regulate the pulmonary fibrosis with cytokines TNF- $\alpha$ and TGF- $\beta$. Clinically recommended medicines for the treatment of pulmonary fibrosis are related to these cytokines. TNF- $\alpha$ can induce the adsorption of inflammatory cells and promote the occurrence of the inflammatory response. It can also regulate the production of other cytokines, deposit collagen, and promote the proliferation of fibroblasts, which plays an important role in the progress of pulmonary fibrosis. TNF- $\alpha$ inhibitors include etanercept, infliximab and adalimumab [48]. TGF- $\beta$ can cause direct and effective stimulation to collagen synthesis, which makes an important impact on the development of pulmonary fibrosis. It takes action by enhancing the activity of connective tissue growth factor (CTGF), promoting the formation of muscle fiber cell type, as well as producing collagen and proteoglycan. Its action mechanism is associated with stimulating the Smads protein pathways. Clinical antifibrotic drugs Pirfenidone can reduce the proliferation of fibroblasts and inhibits collagen synthesis through regulation of TGF- $\beta$ [49]. And some under-developmental TGF- $\beta$ inhibitors such as GC1008, BG00011 and STX-100 can take actions by inhibiting the TGF- $\beta$ pathway [50].

The NF- $\kappa$ B and MAPK pathways are important pathways to cytokine upregulation. The inhibition of these two pathways can reduce cytokines cycle and ease the fibrosis reaction. SP100030 is a type of underdeveloped NF- $\kappa$ B pathway inhibitor. Experiments showed that SP100030 inhibit the protease isomerized to block $\mathrm{NF}-\kappa \mathrm{B}$ pathway and reduces the degree of inflammation and pulmonary fibrosis. Current p38MAPK inhibitors under clinical trials include BIRB796, SB203580, TAK715, etc. BIRB796 shows good inhibition of all p38 MAPK isoforms in-vitro and in vivo [51].

There are no clinical chemokine inhibitors in the treatment of pulmonary fibrosis. Experiments showed that fluorofenidone (a me-too drug of pirfenidone) can inhibit the expression of MCP1 and TNF- $\alpha$ in mice macrophages induced by dead cells, which show the anti-inflammatory action. And fluorofenidone has the influence on NF- $\kappa B$ and MAPK pathway as well [52]. CNTO 888 (Carlumab), an under-developmental human anti-CCL2 antibody, is a monoclonal antibody that binds and neutralizes CCL2, can reduce the migration of macrophages [53]. Binding of CCL2 to its receptor CCR2 triggers chemotaxis. Inhibition of the CCR2 can reduce macrophages migration and accumulation in experimental models [54].

\section{The Effect on AMs and IMs of TCM Treatment of Pulmonary Fibrosis}

Large quantity of experiments showed that TCM had an influence on the expression of cytokines such as TNF- $\alpha$, TGF- $\beta$, PDGF, CTGF, HGF, INF- $\gamma$, etc. [55]. Studies have found that macrophages are main cells which secrete tumor necrosis factor (TNF- $\alpha$ ) in bleomycin-induced pulmonary fibrosis [56]. Experimental results of the buyanghuanwu decoction effect on the AMs of pulmonary fibrosis rats were also found the model group had higher levels of TNF- $\alpha$ than that of the control group. The levels of TNF- $\alpha$ in the model group reached its peak on the $7^{\text {th }}$ day, and fell on the $28^{\text {th }}$ day, with higher levels than the control group. The result showed that TNF- $\alpha$ has an important role in the pathological process of pulmonary fibrosis. The experiment showed that buyanghuanwu decoction can inhibit AMs of lung fibrosis rats to release TNF- $\alpha$. So we can conclude that the mechanism for buyanghuanwu decoction preventing pulmonary fibrosis may be associated with its function of suppressing the TNF- $\alpha$ releasing of AMs [57]. The level of TNF- $\alpha$ in AMs supernatant was measured in each group of different periods during the Lignstrazine study of on pulmonary fibrosis. The results indicated that Lignstrazine could inhibit the release of TNF- $\alpha$ in AM of bleomycin-induced pulmonary fibrosis rats. It can be inferred that the mechanism of Lignstrazine treating pulmonary fibrosis is related to TNF- $\alpha$ release inhibition of AMs [58].

Inthestudy ofdiscussingthe mechanism ofPneumonia Mixture (containing ephedra $60 \mathrm{~g}$ ) in the treatment of pulmonary fibrosis, researchers found that after the formation of bleomycin-induced pulmonary fibrosis in rats, TGF- $\beta 1$ mRNA expressed in AMs and IMs in the model group increased more significantly than that in the control group. Therefore, we can infer that the mechanism of Pneumonia Mixture interfering the pulmonary fibrosis process is possibly associated with the reduction of the TGF- $\beta 1$ expressing 
in AMs and IMs. The main components of Pneumonia Mixture are Astragalus, leeches, Polygonum cuspidatum, etc. Combined treatments of these drugs in all aspects of pulmonary fibrosis remediation inhibit the aggregation of inflammatory cells and reduces the synthesis of TGF- $\beta 1$, as well as reduces abnormal tissues repairing and fibrosis progression [59]. The pathological process of lung diseases including pulmonary fibrosis is called "Xuansu disordered in lung" according to TCM theory. Ephedra and Schisandra are commonly used in the treatment of lung diseases.

Our laboratory has completed ephedra and Schisandra drugcontaining serum on normal rat AMs and IMs secrete TGF- $\beta 1$. In vitro experiments found that ephedra, Schisandra could inhibit the TGF- $\beta 1$ secretion of AMs and IMs. Ephedra-containing serum has a stronger inhibition of AM secretions compared to ephedracontaining serum, whereas Schisandra-containing serum has a stronger inhibition of IM secretions compared to Ephedracontaining serum [60]. The results showed that ephedra and Schisandra may have different influences on pulmonary fibrosis. Ephedra may have a major role in the acute inflammation period while Schisandra may have a major role in pulmonary fibrosis period.

Many experiments also elucidated the mechanism of treating pulmonary fibrosis from the regulation of chemokines. Xuejun $\mathrm{Li}$ showed the concentration of MIP- $1 \alpha$ and MCP- 1 in the plasma of the Panax Notoginseng, the treating group began to rise on the $3^{\text {rd }}$ day and reached the peak on the $7^{\text {th }}$ day in rats of bleomycininduced pulmonary fibrosis, then gradually decreased [61]. The concentration of both MIP- $1 \alpha$ and MCP-1 in the plasma of the treating group had been significantly lower than that of the model group since the $7^{\text {th }}$ day. Zhanshuai Song used Yifei-Huaxian granules in the treatment of paraquat-induced pulmonary fibrosis in rats showed the concentration of MCP-1 reached the peak on the $7^{\text {th }}$ day and kept lower than the model group all through the experiment [62]. The result suggests that Chinese medicine reduces the migration of fibroblasts and inflammatory cells by suppressing the macrophages to express MIP- $1 \alpha$ and MCP-1, thereby reducing the degree of pulmonary fibrosis.

\section{Conclusion}

In conclusion, AMs and IMs are vital regulatory macrophages in the pathological process of pulmonary fibrosis. Because of the anatomical site and cell function of AMs and IMs, anti-fibrosis drug options related to them have higher targeting ability than anti-inflammatory therapy drugs. And AMs and IMs can also be used to explore the multiple mechanisms of traditional Chinese medicine treatment for pulmonary fibrosis.

TCM has made great progress in the experimental study of pulmonary fibrosis prevention. Drugs involving enriching yin and nourishing the blood, clearing up heat and toxin, fortifying the spleen to boost qi, invigorating blood to dissolve stasis, supplementing the lung to boost the kidneys, relieving a cough and panting and many other aspects, meanwhile, the effective components of Chinese medicine provide a larger space and treatment basis for the clinic. Single herb showed good results in fighting against pulmonary fibrosis. At the same time, traditional Chinese medicine decoctions and Chinese patent medicines can have significant effects on pulmonary fibrosis [63,64]. Overall, the mechanisms explanation of the Chinese medicines on pulmonary fibrosis is still in its infancy.

The current studies were largely restricted to animal experiments rather than clinical studies, which focused on anti-inflammatory, immune and cytokines without systematic measurements and standards [65]. Because of the complicated compositions of single Chinese herbs, there is no clear scientific research basis for the effective compositions in the treatment of pulmonary fibrosis. And some therapeutic mechanisms are built on the hypothesis with a lack of rigorous experimental verification. Small samples, different clinical diagnosis and efficacy evaluation criteria result in the no convincing results. Although the research on the molecular and genetic level has been increasing in recent years, it is still limited to a few kinds of Chinese medicines. In this context, some experiments from the perspective of AMs and IMs in the pulmonary fibrosis rats and the influence of some common herbs for lung diseases on the AMs and IMs by using drug-containing serum were conducted. Thus, we can explore the pathological process of pulmonary fibrosis and explain the multiple mechanisms of TCM to guide clinical medication better, which is conducive to the succession and development of traditional Chinese medicine.

\section{Acknowledgement}

This work was supported by the Natural Science Foundation of China (grant numbers 81373887). We are also grateful to Prof. Yang Liu in School of Chinese Pharmacy, Beijing University of Chinese Medicine for providing useful suggestions and assistance.

\section{References}

1. Hutchinson J, Fogarty A, Hubbard R, McKeever T (2015) Global incidence and mortality of idiopathic pulmonary fibrosis: a systematic review. Eur Respir J 46(3): 795-806.

2. Fuqiang Wen, Qianjing Hu (2013) Prospect of the treatment of idiopathic pulmonary fibrosis. Medical Journal of West China 25(1): $1-2$.

3. Shuai Zhang, Dengjia Gang, Huang Sishi, Wenhui Tan (2014) Advances in traditional Chinese medicine treatments of pulmonary fibrosis. Herald of medicine 33(9): 1208-1211.

4. Zisman DA, Keane MP, Belperio JA, Strieter RM, Lynch JP (2005) Pulmonary Fibrosis [M]. Pulmonary Fibrosis. In Fibrosis Research. Totowa, NJ: Humana Press, pp. 3-44.

5. Jun Yang, Qiang Pan, Shiling Wang, Kun Wu (2004) Advances in traditional Chinese medicine treatments of pulmonary fibrosis. China pharmaceuticals 13(8): 21-24.

6. Wenchao Li, Yingjian Zhang, Hong Yang (2014) Based on microRNA expression spectrum technology research progress of pulmonary fibrosis. Journal of Southeast University 33(3): 367-371. 
7. Juan Xue, Weihua Liu, Fei Duan (2014) Research progress of Chinese herb prevention and treatment of pulmonary ifbrosis at the molecular level. Medical Research and Education 31(1): 91-94.

8. Klaus Ley, Alexander Zarbock (2008) From lung injury to fibrosis. Nature Medicine 14(1): 20-21.

9. MS Wilson, TA Wynn (2009) Pulmonary fibrosis: pathogenesis, etiology and regulation. Mucosal Immunol 2(2): 103-121.

10. Lei Han, Tiansong Zhang, Lun Ma, Zixian Zhao (2011) Effect of Buqi Tongfei Yin on Serum Erythrocyte Membrane Fluidity in BleomycinInduced Pulmonary Fibrosis Rats Study. Journal of Traditional Chinese Medicine 19(5): 441-442.

11. Wang XM, Zhang Y, Kim HP, Zhou Z, Feghali-Bostwick CA, et al. (2006) Caveolin-1: a critical regulator of lung fibrosis in idiopathic pulmonary fibrosis. J Exp Med 203(13): 2895-2905.

12. Maeda A, Hiyama K, Yamakido H, Ishioka S, Yamakido M (1996) Increased expression of Platelet-derived growth factor A and insulinlike growth factor-I in BAL cells during the development of bleomycininduced pulmonary fibrosis in mice. Chest 109(3): 780-786.

13. Vanhée D, Gosset P, Boitelle A, Wallaert B, Tonnel AB (1995) Cytokines and cytokine network in silicosis and coal workers' pneumoconiosis Europe Respir J 8(5): 834-842.

14. Baoyu Li, Degang Xiao, Yuhe Liu (1997) The role of alveolar macrophages in the lung fibrosis. Basic \& clinical medicine 17(5): 395397.

15. Lohmann-Matthes ML, Steinmüller C, Franke-Ullmann G (1994) Pulmonary macrophages. Eur Respir J 7(9): 1678-1689.

16. Ravasi T, Wells C, Forest A, Underhill DM, Wainwright BJ, et al. (2002) Generation of diversity in the innate immune system: macrophage heterogeneity arises from the gene-autonomous transcriptional probability of individual inducible genes. J Immunol 168(1): 44-50.

17. Ferrero E, Jiao D, Tsuberi BZ, Tesio L, Rong GW, et al. (1993) Transgenic mice expressing human CD14 are hypersensitive to lipopolysaccharide. Proc Narl Acad Sci USA 90(6): 2380-2384.

18. Wei Zhang, Yaoguang Jiang, Deyu Guo, Chenxiang Hu, Lei Li (2004) Functional and morphological differences between rat alveolar and interstitial macrophages. Am J Respir Cell Mol Biol 26(14): 1242-1244.

19. Yuewen Ma, Xianming Hou, Jian Kang, Yunjiang Yu (2000) TGF $\beta-1$ and its type I, II receptor expression and significance of lung fibrosis. Chinese Journal of Tuberculosis and Respiratory Diseases. 23(11): 5960.

20. Xiaoling Chen, Shansheng Huang, Wenbin Li, Jie Ai (2004) Changes in proliferation and apoptosis of macrophages in the development of pulmonary fibrosis of rats. Chinese Journal of Pathophysiology 20(3): 433-436.

21. Brain JD (1988) Lung macrophages: How many kinds are there? how do they do? Am Rev Respir Dis 137(3): 507-509.

22. Sibille Y, Reynolds HY (1990) Macrophages and polymorphonuclear neutrophils in lung defense and injury. Am Rev Respir Dis 141(2): 471 501

23. Huahua Yin, Jingci Zhu, Lei Li (2004) Functional heterogeneity of monocytes/ macrophages and its significance in immune dysfunction after trauma-hemorrhage in rats. Journal of Third Military Medical University 26(5): 378-381.

24. Huahua Yin, Jingci Zhu, Changguo Gu, Lei Li (2005) Experimental study of heterogeneity in different parts of rat monocytes/macrophage function. Chinese Journal of Cellular and Molecular Immunology 21(4): 422-424.

25. Xiang Guo, Qingqian Yan, Xuebin Zhao, Jingzhi Sun, Zhenglun Wang, et al. (2012) Experimental Study on Toxic Effects to Rat Alveolar
Macrophages by Particulate Matter from Different Chinese Cities. Journal of Environment and Health 29(1): 12-15.

26. Bedoret D, Wallemacq H, Marichal T, Desmet C, Quesada Calvo F, et al. (2009) Lung interstitial macrophages alter dendritic cell functions to prevent airway allergy in mice. J Clin Invest 119(12): 3723-3738.

27. Steinmüller C, Franke-Ullmann G, Lohmann-Matthes ML, Emmendörffer A (2000) Local activation of nonspecific defense against a respiratory model infection by application of interferon- $\gamma$ : Comparison between rat alveolar and interstitial lung macrophages. Am J Respir Cell Mol Biol 22(4): 481-490.

28. Nan Jiang, Daqi Tan, Qiuhua Li, Guixi Liu (2007) Intervention Yiqihuaxian decoction on rats with pulmonary alveolar macrophages and interstitial macrophages TGF- $\beta 1 \mathrm{mRNA}$ expression. Herald of Medicine 26 (10): 1139-1141.

29. Changjun Lv, Xianming Hong (1995) Pulmonary macrophage subsets: alveolar macrophages and interstitial macrophages. Foreign Medical Respiratory 15(4):193-195.

30. Emad A, Emad Y (1995) Relationship Between Eosinophilia and Levels of Chemokines (CCL5 and CCL11) and IL-5 in Bronchoalveolar Lavage Fluid of Patients with Mustard Gas-induced Pulmonary Fibrosis J Clin Immunol 28(6): 298-305.

31. Gaga M, Ong YE, Benyaha F, Aizen M, Barkans J, et al. (2008) Skin reactivity and local cell recruitment in human atopic and nonatopic subjects by CCL2/MCP-1 and CCL3/MIP-1a. Allergy 63(6): 703-711.

32. Lihua Li, Bin Lu, Hongke Wu, Hao Zhang, Feifei Yao, et al. (2015) Role of chemokine receptor CXCR7 in bleomycin-induced lung fibrosis of rat. Modern preventive medicine. 42(23): 4357-4361.

33. Steinman RM, Mellman IS, Muller WA, Cohn ZA (1983) Endocytosis and the recycling of plasma membrane. Cell Biol 96(1): 1-27.

34. Keizer J, Ramirez J, Peacock-López E (1985) The Effect of diffusion on the binding of membrane- bound receptors to coated pits. Biophys 47(1): 79-87.

35. Johnson WJ, Whisnant CC, Adams DO (1981) The binding of BCGactivated macrophages to tumor targets stimulates secretion of the cytolytic factor. J Immunol 127(5): 1787.

36. Pellegrino A, Vacca A, Scavelli C, Dammacco F (2002) Chemokines and tumors. Recenti Prog Med 99(1): 642-654.

37. Xuejun Li, Shehuai Cui, Gencheng Han (2001) Observation of the development of plasma MIP- $1 \alpha$ and MCP-1 in rats with pulmonary fibrosis. Medical Journal of Chinese People's Liberation Army 10(26): 745-747.

38. Yuanyu Zhu, Xiaoling Hu, Yongjian Liu (2002) Losartan in the rat model of bleomycin-induced pulmonary fibrosis and its impact on the expression of monocyte chemoattractant protein- 1 and basic fibroblast growth factor. Zhonghua Jie He He Hu Xi Za Zhi 25(5): 268-273.

39. Teilo H Schaller, Kristen A Batich, Carter M Suryadevara, Rupen Desai, John H Sampson (2017) Chemokines as adjuvants for immunotherapy: implications for immune activation with CCL3. Expert Rev Clin Immunol 13(11): 1049-1060.

40. Zhaokun Shi, Peng Ding, Jie Sun, Yong Shen, Guo Zhao, et al. (2014) Experimental study of monocyte chemoattractant protein-1 for the migration and invasion of macrophage in vitro. Journal of Kunming Medical Universit 35(4): 41-45.

41. Xuewen Lv, Si Zen, Chi Xun Lan (2014) The research progress on role of TGF- $\beta$ and alveolar macrophages in pulmonary fibrosis. Practical Journal of Clinical Medicine 11(2): 9.

42. Xianjiao Cao, Wei Zhang (2017) Review of Study on TCM Intervention in Different Signal Transduction Pathways in Pulmonary Fibrosis in China. Chinese Journal of Chinese Medicine Information 24(2): 127131. 
43. Hua Xiao, Ping Zhang (2008) Transforming Growth Factor-beta Signaling Pathway and Its Therapy of Target in Pulmonary Fibrosis. J Vet Intern Med 8(4): 766-768.

44. Sullivan DE, Ferris M, Pociask D, Brody AR (2008) The Latent Form of TGF $\beta 1$ is Induced by TNF $\alpha$ Through an ERK Specific Pathway and is Activated by Asbestos-Derived Reactive Oxygen Species in Vitro and in Vivo. Journal of Immunotoxicology 5(2): 145-149.

45. Shasha Wang, Bingbing Huan, Wencheng Yu (2016) TRB3, TGF- $\beta$, MAPK signal pathways and pulmonary fibrosis. Journal of Qingdao university medical college 3(52): 373-375.

46. Li Tian, Xian Hua Wang, Xiao Bing Ma, Jing Zhao (2011) Role of tumor nacrosis factor-a signaling pathway mediated by nuclear factor kappa B in pulmonary fibrosis. Modern preventive medicine 38(2): 361-363.

47. Richeldi L, Collard HR, Jones MG (2017) Idiopathic pulmonary fibrosis. Lancet 389(10082): 1941-1952.

48. Yi Du (2015) The pharmacological research and clinical application of the treatment of idiopathic pulmonary fibrosis. Eur Clin Respir J 27(2): 60-63.

49. Poletti V, Ravaglia C, Tomassetti S (2017) Pirfenidone for the treatment of idiopathic pulmonary fibrosis. Expert Rev Clin Pharmacol 10(5): 483-491.

50. Nair GB, Matela A, Kurbanov D, Raghu G (2016) Newer developments in idiopathic pulmonary fibrosis in the era of anti-fibrotic medications. Expert Review of Respiratory Medicine 10(6): 699-711.

51. Kuma Y, Sabio G, Bain J, Shpiro N, Márquez R, et al. (2005) BIRB796 inhibits all p38 MAPK isoforms in vitro and in vivo. J Biol Chem 280(20): 19472-19479.

52. Ling Huang (2013) Study on anti-inflammatory mechanism of fluorofenidone. Central south university.

53. Sandhu SK, Papadopoulos K, Fong PC, Patnaik A, Messiou C, et al (2013) A first-in-human, first-in-class, phase I study of carlumab (CNTO 888), a human monoclonal antibody against CC-chemokine ligand 2 in patients with solid tumors. Cancer Chemother Pharmacol 71(4): 1041-1050.

54. Ekert JE, Murray LA, Das AM, Sheng H, Giles-Komar J, et al. (2011) Chemokine (CC motif) ligand 2 mediates direct and indirect fibrotic responses in human and murine cultured fibrocytes. Fibrogenesis Tissue Repair 4(1): 23.
55. Zhendong Cao, Xianmei Zhou (2013) Progress in the treatment of pulmonary fibrosis related cytokines and the treatment of traditional Chinese medicine. Journal of clinical lung science 18(4): 724-726.

56. Kaelin RM, Center DM, Bernardo J, Grant M, Snider GL (1983) The role of macrophage derived chemoattractant activities in the early inflammatory events of bleomycin-induced pulmonary injury. AM Rev Respir Di 128(1): 132.

57. Fei Wang, Ping Chen, Guoping Cao, Ping Ma (2005) Effects of Buyang Huanwu Decoction (Buyanghuanwutang) on release of tumor necrosis factor from the alveolar macrophage in rats of the bleomycin-induced pulmonary fibrosis. Pharmacology and Clinics of Chinese Materia Medica 21(3): 5-7.

58. Juyuan Liu, Guangfan Hai, Ruili Liu, Xianjun Yang (2005) Effect of Ligustrazine on release of tumor necrosis factor from the experimental pulmonary fibrosis in rats. Journal of Xinxiang Medical College 22(1): $1-3$.

59. Nan Jiang, Daqi Tan, Qiuhua Li (2010) The Intervention of Pneumonia Mixture and Fibrosis in Rat Macrophages and TGF- $\beta 1$ mRNA Expression Changes. Hubei Journal of Traditional Chinese Medicine. 32(8):7-8.

60. Xiaohui Wang (2014) Research of ephedra and Schisandra effect on pulmonary fibrosis based on the idea of translational medicine. Beijing university of Chinese medicine.

61. Xuejun Li, Shehuai Cui (2003) Effects of Panax Notginseng saponin on macrophage inflammatory protein- $1 \alpha$ and monocyte chemoattractant protein-1 in plasma in rats with pulmonary fibrosis. Journal of third military medical university 25(6): 522-524.

62. Zhanshuai Song, Juan Zhang (2016) Effect of Yifei Huaxian granule on TGF- $\beta 1$ and MCP-1 protein expression of rats with paraquat-induced pulmonary fibrosis. Chinese industrial medical journal 29(4): 302-304.

63. Man Qiao, Dongsheng Li (2017) Research on the development of traditional Chinese medicine in idiopathic pulmonary fibrosis. Chinese Journal of Convalescent Medicine 26(4) 367-369.

64. Gaixia Zhao, Xin Zheng (2017) The research of integrated treatment of Chinese and western medicine in the middle and western medicine of idiopathic pulmonary fibrosis. Shandong Chinese medicine journal 36(1): 78-81.

65. Xuan Wang, Xia Li (2016) Traditional Chinese medicine in prevention and treatment of pulmonary interstitial fibrosis. Jilin Chinese medicine 36(9): 966-968.

\section{Your next submission with JuniperPublishers will reach you the below assets}

Commons Attribution 4.0 License DOI: 10.19080/ARGH.2018.10.555790 